\title{
Autogestión del aprendizaje: Revisión de la literatura
}

\section{Learning Self-Management: Literature Review}

Aracelly Fernanda Núñez Naranjo. ${ }^{1}$, Eulalia Beatriz Becerra García. ${ }^{2}$ \& Verónica Elizabeth Olalla Pardo. ${ }^{3}$

\begin{abstract}
.
Introduction. The change in study modality in the last year due to the pandemic, entails an analysis of the different ways in which students can acquire educational knowledge without the constant guidance of the teacher. Objective. The main purpose of this work was to contribute with the construction of definitions on self-management of learning, which include key conceptualizations about self-learning, autonomous learning, directed learning, self-directed learning, self-developed learning, self-management of knowledge, collaborative learning and self-learning regulated. Methodology. The literature search started with 108 documents and by means of inclusion and exclusion criteria, the final studies included were 45. Results. As main results, information was obtained on strategies, actions and tools that allow self-management of learning. Conclusion. It was concluded that self-management of learning refers to four stages, the first forecast, planning and activation, the second monitoring and supervision, the third control and reaction stage, and the fourth reflection and evaluation in which motivation and initiative intervene.
\end{abstract}

Keywords: Learning, Self-management of learning, Autonomy, Motivation

1 Universidad Tecnológica Indoamérica, Carrera de Educación Básica, Ambato, Ecuador. fernandanunez@indoamerica.edu.ec, https://orcid.org/0000-0002-0185-5516.

2 Universidad Tecnológica Indoamérica, Carrera de Educación Básica, Ambato, Ecuador. eulaliabecerra@indoamerica.edu.ec, https://orcid.org/0000-0002-0677-7393.

3 Universidad Tecnológica Indoamérica, Carrera de Educación Básica, Ambato, Ecuador. veronicaolalla@indoamerica.edu.ec, https://orcid.org/0000-0003-3283-5224. 


\section{Resumen.}

Introducción. El cambio de modalidad de estudios en este último año por la pandemia, conlleva un análisis sobre las diferentes formas en las que los estudiantes pueden adquirir conocimientos educativos sin la guía constante del profesor. Objetivo. Este trabajo tuvo como principal propósito aportar con la construcción de definiciones sobre la autogestión del aprendizaje, en la que se incluyen conceptualizaciones claves sobre autoaprendizaje, aprendizaje autónomo, aprendizaje dirigido, aprendizaje autodirigido, aprendizaje auto desarrollado, autogestión del conocimiento, aprendizaje colaborativo y aprendizaje auto regulado. Metodología. La búsqueda de la literatura partió con 108 documentos y mediante criterios de inclusión y exclusión los estudios finales incluidos fueron 45 . Resultados. Como principales resultados se obtuvo información sobre estrategias, acciones y herramientas que permiten autogestionar el aprendizaje. Conclusión. Se concluyó que autogestionar el aprendizaje refiere cuatro etapas, la primera previsión, planificación y activación, la segunda seguimiento y supervisión, la tercera etapa control y reacción, y la cuarta reflexión y evaluación en la que intervienen la motivación y la iniciativa.

Palabras claves: Aprendizaje, Autogestión del aprendizaje, Autonomía, Motivación

\section{Introducción.}

En la actualidad, existe preocupación por aquellos estudiantes que no culminan con éxito sus estudios, o que presentan dificultades en su proceso académico sea en la etapa primaria, secundaria, bachillerato o universitaria, esta situación acarrea factores que diferencian y marcan grupos según el proceso de enseñanza y aprendizaje afectando de manera positiva o negativa a su formación integral y su conocimiento (Núñez, Solano, \& González-Pienda, 2016).

El aprendizaje es un instinto humano que se construye a partir del reconocimiento de diferentes aprendizajes asociados a la conducta y la motivación como experiencia (Cabrera \& Soto, 2020), pues educar requiere dos premisas, la primera es instruir, enseñar y formar (López \& D'Silva, 2020), mientras que la otra supone que el estudiante busca en sí mismo los diferentes medios que tienen como finalidad lograr su propio aprendizaje (Bonilla Olaya, Morales Gualdrón, \& Buitrago Umaña, 2014).

Si bien es cierto que en la actualidad existen diferentes modalidades de estudio, también es cierto que requieren de diferentes factores que permiten y propician en el aprendiz la comprensión desde lo netamente cognitivo pues, lo que se busca es conducirlo y comprometerlo en la elaboración de conocimiento (Bonilla Olaya, Morales Gualdrón, \& Buitrago Umaña, 2014), por medio de participación activa y el desarrollo de su independencia cognitiva (Calcines Castillo, Valdés Utrera, \& Monteagudo de la Guardia, 2017). 
Gestionar el propio aprendizaje, implica un proceso complejo (Valiente-Barroso, SuárezRiveiro, \& Martínez-Vicente, 2020) en el que intervienen factores asociados a aspectos internos como: el personal, familiar, económico, y otros externos como: los institucionales y académicos que influyen en la vida de los estudiantes (Núñez-Naranjo, Ayala-Chauvin, \& Riba-Sanmartí, 2021) pues conlleva a contextos diferenciados que permiten o impiden gestionar su tiempo dedicado al estudio.

En un concepto pedagógico esta autogestión del aprendizaje se relaciona con otros conceptos investigados por varios autores como son: aprendizaje dirigido, aprendizaje auto dirigido, aprendizaje auto desarrollado, aprendizaje auto regulado, auto gestión del conocimiento, aprendizaje autónomo entre otros (Azpilicueta, 2020; Balbás, GarcíaBerrocal, Montalvo, \& Díaz de Villafranca, 2011; Calcines Castillo, Valdés Utrera, \& Monteagudo de la Guardia, 2017), que tienen como propósito (Barría, Rodríguez, \& Salmerón, 2017) la independencia, responsabilidad y motivación por aprender y prepararse. La autogestión del aprendizaje se entiende como aquella en la que el estudiante planifica, controla y evalúa su propio aprendizaje por medio del cumplimiento de objetivos académicos y motivacionales haciendo uso de recursos materiales, tecnológicos y humanos (Góngora, 2005).

Los hábitos de estudio, involucran autonomía y una decisión del individuo sobre su criterio propio a prepararse por medio de gestionar y priorizar su tiempo adaptándose a un contexto educativo autónomo (Balbás, García-Berrocal, Montalvo, \& Díaz de Villafranca, 2011), esta situación se integra a la idea de saber aprender para aprender a aprender controlando su propio aprendizaje (Villardón \& Yániz, 2011). La autogestión del aprendizaje requiere del uso de diferentes procesos cognitivos, motivacionales, meta cognitivos, conductuales y afectivos que tienen como propósito la construcción de un nuevo conocimiento o el fortalecimiento de uno anterior (Chaves Barboza, 2014; Valiente-Barroso, Suárez-Riveiro, \& Martínez-Vicente, 2020; Pérez \& Castejón Costa, 2008).

La integración de elementos cognitivos y motivacionales se encuentran directamente relacionados, siendo necesarios para una visión general del aprendizaje, pues debe existir una relación entre las estrategias meta cognitivas y cognitivas con la motivación, la conducta y la iniciativa de los estudiantes sobre sus actividades académicas (Pérez \& Castejón Costa, 2008; Calcines Castillo, Valdés Utrera, \& Monteagudo de la Guardia, 2017), dicho de esta manera, en esta interacción intervienen diferentes etapas (Barría, Rodríguez, \& Salmerón, 2017). Auto-gestionar el aprendizaje refiere cuatro etapas, la primera se conoce como previsión, planificación y activación, la segunda es la etapa del seguimiento y supervisión, la tercera trata sobre el control y reacción, y la cuarta sobre la reflexión y evaluación (Chaves Barboza, 2014; Barría, Rodríguez, \& Salmerón, 2017). A continuación, se nombran algunas definiciones entorno al campo temático de estudio.

Autoaprendizaje.- Monereo, Castelló, Clariana, Palma, \& Pérez (1999) mencionan que el autoaprendizaje es el proceso mediante el cual el estudiante adquiere conocimientos teóricos y técnicos, sin ayuda directa, indirecta o intencionada (Estrada, Fuentes, \& 
Blanco, 2018), Vargas (2002) por su parte lo define como aquella facultad que le permite al individuo a tomar decisiones y dirigir su propio aprendizaje en función de un objetivo y un contexto determinado, mientras que Birembaum (2002), establece que el autoaprendizaje puede conducir a un mayor logro académico e incrementar la eficacia y eficiencia y que los cambios se pueden ver reflejados en una conducta más autorregulatoria, en la que se involucran los procesos sociales e interpersonales y los procesos individuales.

Aprendizaje autónomo.- Para Villavicencio (2004) y Solórzano-Mendoza (2017) el aprendizaje autónomo es aquel en el que una persona tiene la capacidad para dirigir, controlar, regular y evaluar su manera de aprender haciendo uso de estrategias de aprendizaje, procedimientos, recursos, con un rol activo y de forma consciente e intencionada.

Aprendizaje dirigido.- Para Hernandez, Dominguez \& Caballero (2005), el aprendizaje dirigido se concibe de acuerdo al impacto de adquisiciones de conocimiento, en el que se involucra a la motivación e iniciativa propia que se ejerce por la necesidad de un aprendizaje nuevo en el que intervienen métodos con los que el estudiante va desarrollando una de las características humanas para un aprendizaje permanente y continuo (Savater, 2003).

Aprendizaje autodirigido.- Ross (1999), establece que el aprendizaje autodirigido enfoca a un gran número de interpretaciones que se movilizan de acuerdo al lugar y al espacio en el que se estudie o trate de comprenderse, en este se incluyen tanto habilidades, cómo técnicas y procedimientos por medio de las que se determinan las diferentes metas y objetivos, se localizan los recursos, se plantea estrategias y se evalúan los resultados. El aprendizaje autodirigido para Fasce y otros (2013), es aquel en el que el estudiante está en la capacidad de dirigir y diagnosticar su proceso de aprendizaje, sin embargo, se requiere de competencias y orientaciones que motiven al estudiante al logro de su meta.

Aprendizaje auto desarrollado. - Según Torrano (2004) el aprendizaje auto desarrollado, se logra de un aprendizaje significativo y autorregulado por tanto necesita voluntad como destreza para poder ejercer la acción para enseñar y aprender. Este aprendizaje busca que los individuos sean conscientes de su pensamiento y sean estratégicos para que puedan dirigir su motivación hacia sus metas, es decir busca que los individuos aprendan a ser sus propios maestros.

Autogestión del conocimiento.- Según Martínez (2014) define a la Autogestión del conocimiento, como los conocimientos acumulados de forma individual y colectiva constituyen fuente para estructurar cualquier forma de superación, esta autogestión involucra una decisión personal en la que el aprendiz como dueño de aprendizaje es capaz de controlar, monitorear y evaluar sus objetivos, metas académicas y su motivación además de administrar de manera correcta los recursos tanto humanos como materiales.

Aprendizaje colaborativo. - Para Salmons (2008) el aprendizaje colaborativo es aquel en el que el conocimiento se construye a base de la negociación entre los diferentes 
significados y la solución de los problemas reales en los diferentes contextos en los que el aprendiz interactúa con otros. El trabajo colaborativo realizado mediante las herramientas informáticas permite la construcción y vencimiento de barreras entorno a la comunicación, comprensión y ejecución de liderazgos con el propósito de llevar a cabo una construcción equitativa del conocimiento (Hernández, 2014).

Aprendizaje auto regulado. - Para Zulma (2006) el aprendizaje autorregulado refiere la metacognición que tiene como finalidad un aprendizaje exitoso, en el que se asume una distinción de procesos sumamente difusa, observando ambigüedad y superposición entre estos aspectos. El aprendizaje autorregulado durante la adquisición de conocimientos o destrezas es un proceso complejo a través del cual los individuos propician un control sobre su propio pensamiento y en el que interviene tanto el afecto como la conducta, las que permitirán una mayor atención ante la enseñanza impartida permitiendo tener una noción racional de la actividad que se está ejecutando (Contreras \& Lozano, 2012).

\section{Metodologia.}

Búsqueda de la literatura. - Se identificaron estudios relevantes sobre autogestión del aprendizaje, y publicaciones con similares contenidos como aprendizaje dirigido, aprendizaje autónomo, aprendizaje auto regulado, aprendizaje auto dirigido, auto gestión del conocimiento, auto gestión del aprendizaje partiendo del trabajo realizado por los autores como Durlak \& Lipsey (1991).

Se realizó una búsqueda bibliográfica sistémica con base de datos en SCOPUS, SCIENCIE, LATINDEX, REDEBID, SCIELO, en la que se utilizó múltiples palabras clave. Se procedió con una búsqueda adicional de las citas de las publicaciones incluidas en este trabajo para ello se utilizó buscadores como google scholar, publish or-perich con la finalidad de detectar artículos científicos disponibles.

Los artículos científicos fueron examinandos mediante la lectura del resumen ejecutivo o abstract, con el principio de inclusión y exclusión. Se partió con una base de datos de 108 documentos de los que en base a la información de su resumen ejecutivo y con principios de inclusión se dio lectura a todo el documento. La búsqueda sistémica bibliográfica finalizó en febrero de 2021.

Criterios de Inclusión y exclusión. - Los artículos que se examinaron, 1: partiendo del criterio de inclusión entorno a la autogestión del aprendizaje, 2: los documentos se centran en campo temático de estudio, 3: los documentos incluyen fases, etapas, paradigmas y estrategias utilizadas, 4: los documentos cuentan con información sobre aprendizaje dirigido, aprendizaje autónomo, aprendizaje auto regulado, aprendizaje auto dirigido, auto gestión del conocimiento, auto gestión del aprendizaje. Existieron estudios que se excluyeron porque 1: la información contenida no aportaba para la revisión sistémica, 2: no se aclaraba las fases o etapas o paradigmas, y 3: el contenido tenía un enfoque diferente al requerido para este trabajo. 
Codificación del trabajo. - Los documentos incluidos tuvieron diferentes enfoques sobre las etapas, el método y las estrategias utilizadas. Ya que las investigaciones tuvieron una heterogeneidad, se excluyeron e incluyeron en concordancia con el campo temático y sus palabras claves similares que fueron definidos en los documentos

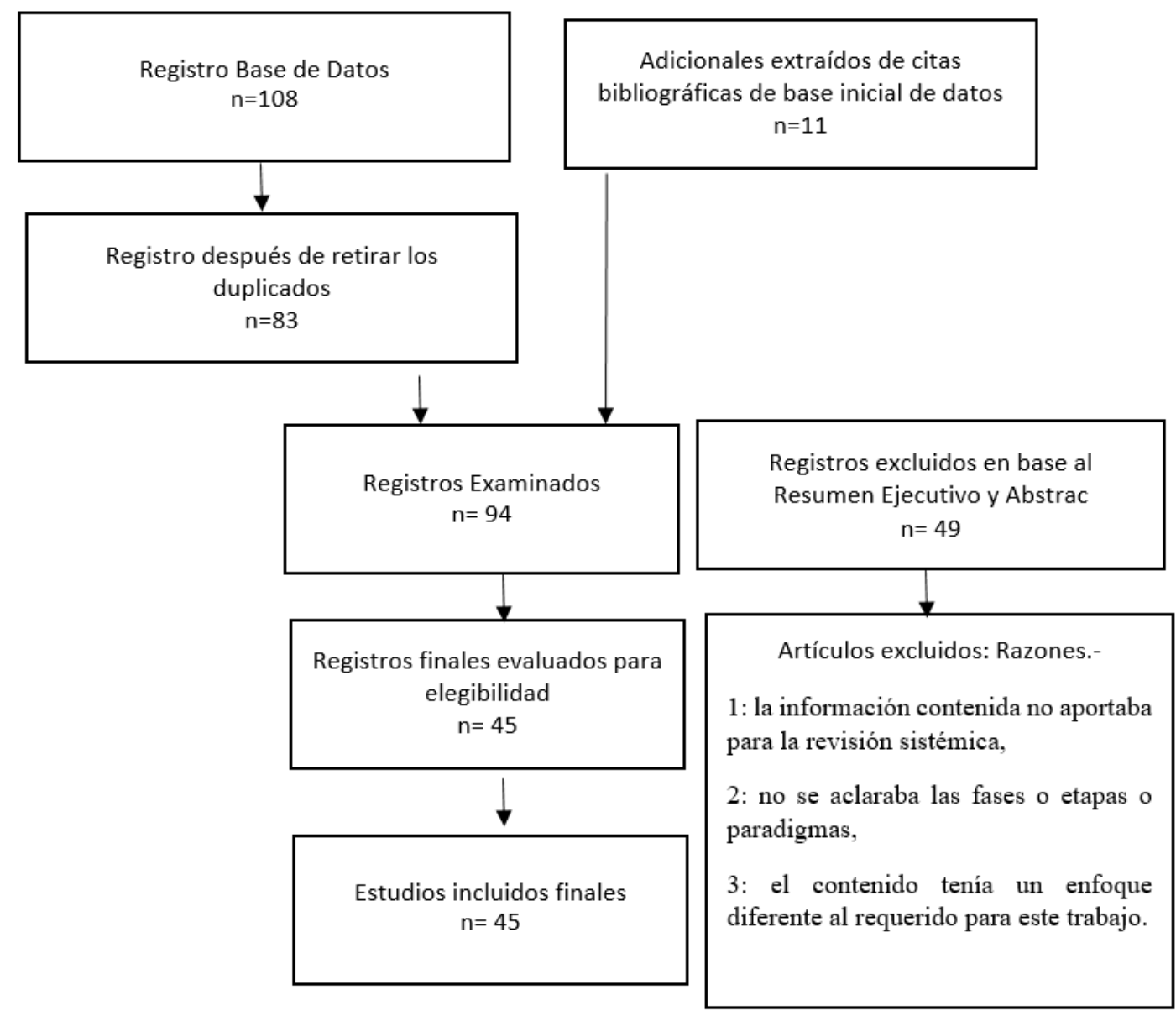

Gráfico No. 1:

Diagrama de flujo de artículos seleccionados

\section{Resultados.}

Para que los estudiantes logren autogestionar su aprendizaje, se requiere de estrategias que desarrollen la autonomía e iniciativa y den pautas para que el educando fortalezca tanto la cognición como la motivación (Fernández Cueli, García, \& González-Castro, 2013; Calcines Castillo, Valdés Utrera, \& Monteagudo de la Guardia, 2017), mejorando el rendimiento académico.

Estrategias para la autogestión del aprendizaje: Para lograr la autogestión del aprendizaje autores como (Valiente-Barroso, Suárez-Riveiro, \& Martínez-Vicente, 2020) mencionan que para obtener datos relevantes entorno a la temática se aplican estrategias de apoyo, en las que se deben considerar el tiempo, lugar, materias de estudio, motivación, 
concentración, iniciativa y relajación y estrategias complementarias consideradas adicionales tales como actividades extra escolares, trabajos en grupo, apuntes, uso de recursos y materiales

Principales acciones de autogestionar el aprendizaje: Anteriormente, el aprendizaje se veía como aquel en el que el profesor era directamente el responsable de los saberes de sus estudiantes, sin embargo, en la actualidad el aprendizaje es visto desde otra perspectiva en la que intervienen saberes previos, metas académicas, auto-concepto, conducta, actitudes, aptitudes, expectativas, estrategias entre otras que involucran la motivación (Miñano Pérez \& Castejón Cost, 2008) las que pueden incidir en el quehacer pedagógico. Si bien es cierto gestionar el aprendizaje requiere de motivación propia y predisposición, lograr el conocimiento de los estudiantes involucra una retroalimentación adecuada, motivadora y positiva debido a que integra no solo memorización o procedimientos para la resolución de problemas sino el desarrollo y fortalecimiento de habilidades como el pensamiento crítico, comunicación asertiva, autoestima, autogestión y motivación (Azpilicueta, 2020), en el que tanto el profesor como el estudiante interactúan con herramientas didácticas en la que la propuesta pedagógica es flexible y abierta (Bonilla Olaya, Morales Gualdrón, \& Buitrago Umaña, 2014).

Aprendizaje con herramientas tecnológicas, existen varios estudios que relacionan a la autogestión del aprendizaje con el uso de las TIC's, con la educación a distancia, online o hibrida, o con el desarrollo de competencias del aprendizaje autónomo, sin embargo, esta autogestión del aprendizaje enfatiza momentos del aprendizaje y una reflexión en torno al lado personal y la relación con su entorno inmediato (Cabrera \& Soto, 2020; Bruna Jofré, Bunster Balocchi, Martínez Oyanedel, \& Márquez Urrizola, 2014; López \& D'Silva, 2020). Por lo general, al tratar sobre el aprendizaje en estas modalidades conlleva a pensar en una serie de herramientas y plataformas tecnológicas que con el establecimiento de estrategias didácticas se emplean para una mediación pedagógica, la posibilidad de trabajar cooperativamente, acceso a la información de manera más eficiente, recursos multimediales y audiovisuales entre otros (Calderón-Meléndez, 2020; Barreto, 2011).

Las diferentes acciones que llevan a los estudiantes a autogestionar su aprendizaje en este tiempo de pandemia, han sido en la educación superior apoyadas por la tecnología sostenido y evolucionado con el tiempo, de esta manera el docente asume un rol de guía, estimulador y colaborador en el proceso de aprendizaje del estudiante. La integración y desarrollo de diferentes softwares han generado oportunidades en las que no se requiere la presencialidad abriendo puertas a la posibilidad de aprender de manera autónoma, este escenario genera la posibilidad de que por medio de la utilización de estrategias gestiones su propio aprendizaje mejorando su rendimiento académico (Cerda, López, Osses, \& Saiz, 2015). 


\section{Discusión.}

- Bandura (1983), menciona que gestionar el aprendizaje está ligado a la motivación y la suficiencia para el establecimiento de metas deseadas, de la misma manera Chavez \& Morales (2020), establecen que es el aprendiz, en su iniciativa y autonomía el que decide aprender y en qué grado, Valdés \& Pujol (2012) por su parte, consideran que el eje central para autoaprendizaje está orientado a actividades que logren además el desarrollo de la autorregulación, Monereo \& Pozo (2007) indican que gestionar el aprendizaje refiere también a una serie de competencias concretas que favorecen la autonomía, sin embargo para Martínez \& Pool (2013) este conjunto de competencias relacionadas son consideradas positivas solo cuando la autoeficacia es percibida y las metas de aprendizaje logradas, visto de esta manera la motivación se involucra en todo el entorno aprendizaje debido a que está ligado directamente con la disposición del alumno y su interés en el que se involucran componentes motivacionales como la planificación, la activación motivacional, el control y regulación de la motivación (Valle, Rodríguez, Núñez, \& Cabanach, 2010).

- La autogestión del aprendizaje según Cerda (2012) está definida como la participación activa del estudiante en el diseño, la conducción y la evaluación de un esfuerzo por aprender, para Estrada, Fuentes, \& Blanco (2018) este aprender se realiza de manera autónoma asumiendo la responsabilidad sobre planificar, controlar y evaluar lo aprendido, mientras que Chaves Barboza (2014) menciona que la autogestión del aprendizaje se puede presentar desde varias aristas en las que intervienen de manera indirecta el docente y en el que además se relaciona la investigación educativa que puede tener un tinte empírico sobre los entornos personales de aprendizaje, para Monereo, Castelló, Clariana, Palma, \& Perez (1999) esta autogestión es útil una vez se cumplan con el objetivo de aprender de manera autónoma involucrando diferentes áreas del aprendizaje activo en el que el individuo esté en la capacidad de integrar, participar, actuar y adquirir información produciendo un conocimiento más amplio y duradero.

- Promover la autogestión para el aprendizaje es una tarea conjunta entre las instituciones educativas y el individuo pues se consideran indispensable para una mejor adquisición de saberes; el uso creciente de la tecnología permite mediar los procesos de formación que son necesarios para que el sujeto que aprende tome las riendas de su trayecto educativo y active otras capacidades asociadas, como la criticidad, el análisis y la colaboración en el que el intervienen cuatro etapas, previsión, planificación y activación, seguimiento y supervisión, el control y reacción, la reflexión y evaluación. 


\section{Referencias bibliográficas.}

Acurio Ponce, B., \& Nuñez Naranjo, A. (2019). Creo, juego y aprendo con estrategias y recursos para mejorar la comprensión lectora. 593 Digital Publisher CEIT, 4(2), 44-59. doi:https://doi.org/10.33386/593dp.2019.2.87

Alonso, J., \& Mateos, M. (1985). Comprensión lectora: Modelos entrenamiento y evaluación. Infancia y Aprendizaje, 5-19. Obtenido de https://media.utp.edu.co/referencias-

bibliograficas/uploads/referencias/articulo/27-comprension-lector-modelosentrenamiento-evolucion-infancia-y-aprendizajepdf-fnUv0-articulo.pdf

Anaya-Durand, A., \& Anaya-Huertas, C. (2010). ¿Motivar para aprobar o para aprender? Estrategias de motivación del aprendizaje para los estudiantes. Tecnológia, $\begin{array}{llll}\text { ciencia } y & \text { educación, } & \text { Obtenido de }\end{array}$ https://www.redalyc.org/pdf/482/48215094002.pdf

Aquino, R. (2020). El Cuento: Estrategia Docente para la Comprensión Lectora. Obtenido http://tesis.usat.edu.pe/bitstream/20.500.12423/2646/1/TL_AquinoZapataRosa.p df

Asamblea Nacional Constituyente del Ecuador. (2008). Constitución de la República del Ecuador.

Asamblea Nacional de la República del Ecuador. (2010). LOES. En LEY ORGANICA DE EDUCACION SUPERIOR, LOES. Quito: Presidencia de la Republica del Ecuador.

Azpilicueta, M. (2020). LOS BENEFICIOS DE UNA CORRECTA EVALUACIÓN FORMATIVA EN EL AUTOAPRENDIZAJE DE LOS ALUMNOS. JOURNAL OF SUPRANATIONAL POLICIES OF EDUCATION, 12, 2-25. doi:: https://doi.org/10.15366/jospoe2020.12.001

Balbás, M., García-Berrocal, A., Montalvo, C., \& Díaz de Villafranca, J. (2011). UNA EVALUACIÓN ON-LINE A LA DEMANDA PARA EL AUTOAPRENDIZAJE. ARBOR Ciencia, Pensamiento y Cultura, 243-248. doi:doi: 10.3989/arbor.2011.Extra-3n3152

Bandura. (1983). Mecanismos de autoevaluación y autoeficacia que gobiernan los efectos motivacionales de los sistemas de objetivo. Investigacion cientifica, 8-15. 
Barreto. (2011). Desarrollo de competencias interculturales en ambientes virtuales de aprendizaje. "Revista Virtual Universidad Católica del Norte”. doi:ISSN 01245821

Barría, C., Rodríguez, S., \& Salmerón, P. (2017). Autorregulación del aprendizaje en centros educativos de Granada donde se utilizan las Tecnologías de la Información y la Comunicación. REIDOCREA, 140-155. doi:ISSN: 2254-5883

Benavides, D. (13 de Mayo de 2013). Estrategias Didácticas para fomentar la Lectura Crítica . Obtenido de https://www.redalyc.org/pdf/551/55128038004.pdf

Bermúdez Jiménez, D., Casanova Ropero, K., \& Villarreal, E. A. (2015). Estrategias motivacionales dirigidas a estudiantes del ciclo básico, inscritos e la carrera de contaduría pública de la Facultad de Ciencias Económicas y Sociales de la Universidad de Carabobo.

Birembaum, M. (2002). Assesing self-directed active learning in primary schools. Assessment in Education: principles, Policy and Practics, , 32-33. doi:1665-0824

Bonilla Olaya, J., Morales Gualdrón, L., \& Buitrago Umaña, E. (2014). Modelo pedagógico para el aprendizaje en red basado en el constructivismo sociocultural: una alternativa para la apropiación de conocimiento en América Latina. Equidad \& Desarrollo(21), 163-185. doi: ISSN 1692-7311

Bruna Jofré, C., Bunster Balocchi, M., Martínez Oyanedel, C., \& Márquez Urrizola, C. (2014). Utilizar la wiki para promover autoaprendizaje y responsabilidad social en futuros científicos. Educación Médica Superior, 28(2), 229-242. Obtenido de http://scielo.sld.cu/scielo.php?script=sci_arttext\&pid=S086421412014000200005

Cabrera, V., \& Soto, C. (2020). ¿CÓMO APRENDEMOS? EL DOCENTE ENSEÑANTE Y APRENDIZ QUE ACOMPAÑA A LOS ESTUDIANTES EN SU EXPLORACIÓN HACIA EL (AUTO)APRENDIZAJE. Revista y Curriculum del Profesorado, 24(3), 270-290. doi:10.30827/profesorado.v24i3.8155

Calcines Castillo, M., Valdés Utrera, J., \& Monteagudo de la Guardia, R. (2017). La autogestión del conocimiento: ¿una meta inalcanzable? EDUMECENTRO, 9(2), 225-229. doi:ISSN 2077-2874

Calderón-Meléndez, A. (2020). Elementos clave de la virtualidad en la educación superior. REVISTA ELECTRÓNICA CALIDAD EN LA EDUCACIÓN SUPERIOR, 11(2), 80-104. doi:DOI: http://dx.doi.org/10.22458/caes.v11i2.3322

Carranza, L. (2018). Factores que determinan la Falta de Motivación hacia la Lectura. Obtenido 
http://repository.ut.edu.co/bitstream/001/2466/1/T\%200945\%20601\%20CD600 4\%20APROBADO\%20LUIYIS\%20ELIGIO\%20CARRANZA\%20RUIZ.pdf

Carrasco Altamirano, A. (2003). La escuela puede enseñar estrategias de lectura y promover su regular empleo. Revista Mexicana de Investigación Educativa, 128142. Obtenido de https://www.redalyc.org/pdf/140/14001708.pdf

Cerda, C., \& Osses, S. (2012). Aprendizaje autodirigido y aprendizaje autorregulado: dos conceptos diferentes. Mpedica Chile, 140(11), 6-15. doi:http://dx.doi.org/10.4067/S0034-98872012001100020

Cerda, C., López, Ó., Osses, S., \& Saiz, J. (2015). Análisis Psicométrico de la Escala de Aprendizaje de Autodirigido Basada en la Teoría de Aprendizaje Autodirigido de Garrison. Revista Iberoamericana de Diagnóstico y Evaluación, 1(39). doi:ISSN: $1135-3848$

Contreras, Y., \& Lozano, A. (2012). Aprendizaje auto-regulado como competencia para el aprovechamiento de los estilos de aprendizaje en alumnos de educación superior. Revista Estilos de Aprendizaje, $n^{\circ} 10$, Vol 5, 5(10), 114-147. Obtenido de http://revistaestilosdeaprendizaje.com/article/view/964/1672

Chaves Barboza, E. (2014). Autogestión del aprendizaje en la investigación educativa sobre Entornos Personales de aprendizaje (PLE): Una revisión de literatura. EDEMIC, 3(2), 114-134. doi:https://doi.org/10.21071/edmetic.v3i2.2892

Chavez, J., \& Morales, M. (2020). Educación en línea: análisis del aprendizaje autodirigido en estudiantes de posgrado. Revista Electrónica sobre Tecnologia, Educación y Sociedad, 14, 10-11. doi:ISSN: 2448-6493

Durlak, J., \& Lipsey, M. (1991). A practitioner's guide to meta-analysis. Am J Commun Psychol, 19, 291-332. doi:https://doi.org/10.1007/BF00938026

Estrada, O., Fuentes, D., \& Blanco, S. (2018). ESTRATEGIA PARA LA FORMACIÓN PROFESORAL EN EL AUTOAPRENDIZAJE ESTUDIANTIL. Opuntia Brava, 10(4), 7-8. doi:ISSN: 2222-081x

Fasce, E., Ortega, J., Perez, C., Marquez, C., Parra, P., Ortiz, L., \& Matus, O. (2013). Aprendizaje autodirigido en estudiantes de primer año de medicina de la Universidad de Concepción y su relación con el perfil sociodemográfico y académico. Scielo(9), 1117-1125. doi:http://dx.doi.org/10.4067/S003498872013000900003

Fernández Cueli, M., García, T., \& González-Castro, P. (2013). Autorregulación y rendimiento académico en Matemáticas. Dianet, 41(1), 39-48. doi: ISSN 02102773 
Ferreri, E. (2015). Estrategias compensatorias en el proceso de lectura de una LE: un recorrido teórico hacia una implementación práctica. Universidad Tecnológica Nacional. Obtenido de https://fcf.unse.edu.ar/eventos/eici2015/contenido/pdf/02.pdf

Flores Guerrero, D. (2016). La importancia e impacto de la lectura, redacción y pensamiento crítico en la educación superior. Redalyc, 128-135. Obtenido de https://www.redalyc.org/pdf/853/85346806010.pdf

Góngora, J. J. (2005). La autogestión del aprendizaje en ambientes educativos centrados en el alumno. Boletín del Modelo Educativo, Tecnología de Monterrey. Obtenido de http://www. sistema. itesm. mx/va/dide/botetin_9/pag, 3.

Hernandez, J., Dominguez, M., \& Caballero, M. (2005). Innovación de producto y aprendizaje dirigido en alfarería en Oaxaca, México. Scielo, 11(2), 213-228. Obtenido de http://ve.scielo.org/scielo.php?pid=S131595182005000200002\&script=sci_arttext

Hernández, N. G. (2014). La planificación del aprendizaje colaborativo en entornos virtuales. Comunicar. doi:0.3916/C42-2014-02

Hoyos Flórez, A. M., \& Gallego, T. M. (2017). Desarrollo de habilidades de comprensión lectora en niños y niñas de la básica primaria. Revista Virtual Universidad Católica del Norte(51), 23-45. Obtenido de https://www.redalyc.org/pdf/1942/194252398003.pdf

Jiménez Ortíz, M., \& Gordo Contreras, A. (2014). EL CUENTO INFANTIL: FACILITADOR DE PENSAMIENTO DESDE UNA EXPERIENCIA PEDAGÓGICA. 151-170. doi:ISSN 2216-0159

Jouini, K., \& Saud, K. (2005). ESTRATEGIAS INFERENCIALES EN LA COMPRENSIÓN LECTORA. Revista de educacion, 96-115. Obtenido de http://www.aldadis.net/revista4/04/imagen/04jouini.pdf

Kistemaker, M., \& Broeder, P. (2014). Fremdsprachenunterricht im Spannungsfeld zwischen Sprachwissen und Sprachkönnen. Saarbrücken: Thomas Tinnefeld. doi:https://www.academia.edu/10232366/School_language_and_the_role_of_m ultilingualism_in_class

López, C., \& D'Silva, F. (2020). Enseñar en pandemia: Diseño Instruccional (DI) como herramienta fundamental para atreverse en la educación digital. Rediunp, 2(1), 321.

Obtenido de http://www.revistas.unp.edu.ar/index.php/rediunp/article/view/158/129

Marina, L. (2014). La Comprensión Lectora Desde El Contructivismo. 
Marínez Chepe, A., Tocto, C., \& Palacios, L. (2015). La expresión oral en los niños y los cuentos. UCV HAcer, Revista de Investigación y Cultura, 116-120. doi:ISSN: $2305-8552$

Martínez, \& Pool. (2013). Autoeficacia y uso de estrategias para el aprendizaje. Revista Electrónica de Investigación Educativa, 3.

Martínez, G. (2014). La preparación de los docentes en la utilización de los materiales didácticos. Obtenido de https://www.eumed.net/rev/atlante/2018/09/docenteformador-maestros.html

Mendoza Velazco, D. J. (2018). Estrategias didácticas para el fortalecimiento del proceso lector a nivel andragógico. UIDE, Universidad Internacional del Ecuador, 35-52. Obtenido de https://revistas.uide.edu.ec/index.php/innova/article/view/403

Miñano Pérez, P., \& Castejón Cost, J. (2008). Capacidad predictiva de las variables cognitivo-motivacionales sobre el rendimiento académico. Revista Electrónica de Motivación y Emoción, 9(28). Obtenido de http://reme.uji.es/articulos/numero28/article4/article4.pdf

Monereo, C., \& Pozo, J. (2007). Competencias para (con) vivir con el siglo XXI. Cuadernos de pedagogía, 370(12), 12-18. Obtenido de http://maaz.ihmc.us/rid=1GLSWYC7N-Y2LLHHH7H/Competencias\%20para\%20convivircon\%20el\%20siglo\%20XXI.pdf

Monereo, C., Castelló, M., Clariana, M., Palma, M., \& Perez, L. (1999). Estrategias de enseñanza y aprendizaje. Formación del profesorado y aplicación en la escuela. Barcelona: Grao. Obtenido de ttps://d1wqtxts1xzle7.cloudfront.net/56174095/RESUMEN_DE_ESTRATEGIA S_DE_ENSENANZA_Y_APRENDIZAJE_DE_MONEREO.pdf?1522169295= \&response-content-

disposition=inline\%3B+filename\%3DEstrategias_de_ensenanza_y_aprendizaje. pdf $\&$ Expires $=1616793834 \&$ Signature $=$ guitcduVJJ

Núñez, J., Solano, P., \& González-Pienda, J. (2016). Evaluación de los procesos de autorregulación mediante autoinforme. Psicothema, 353-358. doi:ISSN 0214 9915

Núñez-Naranjo, A., Ayala-Chauvin, M., \& Riba-Sanmartí, G. (2021). Prediction of University Dropout Using Machine Learning. ICITS 2021., 396-406. doi:https://doi.org/10.1007/978-3-030-68285-9_38

Ospina Rodríguez, J. (2006). La motivación, motor del aprendizaje. Revista Ciencias de la Salud, 158-160. Obtenido de https://www.redalyc.org/pdf/562/56209917.pdf

Padial Ruz, R., \& Sáenz-López, p. (2013). Los cuentos populares/tradicionales en educación infantil. Una propuesta a través del juego. Revista de Educación, 


Motricidad $\quad e \quad$ Investigación, 32-47. Obtenido de
https://www.researchgate.net/profile/Rosario_Padial_Ruz/publication/33167541 1_LOS_CUENTOS_POPULARESTRADICIONALES_EN_EDUCACION_IN FANTIL_UNA_PROPUESTA_A_TRAVES_DEL_JUEGO/links/5cbda076a6fd cc1d49a62e58/LOS-CUENTOS-POPULARES-TRADICIONALES-ENEDUCACION-INFANTI

Parra Delgado, G. (2008). Cuento colectivo: El Castillo. Innovación y experiencias educativas, 1-13. Obtenido de https://archivos.csif.es/archivos/andalucia/ensenanza/revistas/csicsif/revista/pdf/ Numero_12/GEMA_PARRA_1.pdf

Pérez, P., \& Castejón Costa, J. (2008). Capacidad predictiva de las variables cognitivomotivacionales sobre el rendimiento académico. Revista Electrónica de Motivación $y \quad$ Emoción, XI(28), 1-13. Obtenido de http://reme.uji.es/articulos/numero28/article4/article4.pdf

Prieto, T. (17 de Enero de 2021). Actividades y estrategias de comprensión lectora en las aulas. Obtenido de http://www.jstor.org/stable/23766966

Ramírez Leyva, E. M. (2009). ¿Qué es leer? ¿Qué es la lectura?. Investigación bibliotecológica,. Scielo, 161-188. Obtenido de Recuperado en 15 de enero de 2021, de http://www.scielo.org.mx/scielo.php?script=sci_arttext\&pid=S0187$358 X 2009000100007 \& \operatorname{lng}=$ es\&tlng=es.

Redondo Gonzalez, A. (2008). La comprensión lectora. Innovación y experiencias educativas, 1-8. doi:ISSN: 1988-6047

Rojas Camargo, G., \& Prieto Martínez, M. (2017). El cuento multimodal como strategia didáctica para el aprendizaje de la lectura. Educación y Ciencia, 297-312. Obtenido https://revistas.uptc.edu.co/index.php/educacion_y_ciencia/article/view/10286

Ross. (1999). Self-directed learning readiness among graduate students: Implications for Orientation Program. Journal of College Student Development, 739.

Salmons, J. E. (2008). Taxonomy of Collaborative E-learning. Encyclopedia of information technology curriculum integration. doi:1138-414X

Savater, F. (2003). El valor de elegir. Colombia: Ariel. Obtenido de https://www.redalyc.org/pdf/311/31161105.pdf

Serrabona, J. (2008). Los cuentos vivenciados: imaginación y movimiento. Facultat de Psicologia, Ciències de l'Educació i de L'Esport Blanquerna, 61-78. doi:ISSN 0213-8646 
Solórzano-Mendoza, Y. (2017). Aprendizaje autónomo y competencias. Revista Científica Dominio de las Ciencias, 241-253. Obtenido de https://dialnet.unirioja.es/servlet/articulo?codigo $=5907382$

Thomson, N. (2012). Language Teaching Strategies and Techniques Used to Support Students Learning in a Language other than Their Mother Tounge. Kongsberg International School. Obtenido de https://ibo.org/contentassets/4ccc99665bc04f3686957ee197c13855/thompson_e xecsum_8-29-12.pdf

Tirado, F., Santos, G., \& Tejero-Díez, D. (2013). La motivación como estrategia educativa: Un estudio en la enseñanza de la botánica. Scielo, 39(139), 79-92. Obtenido de http://www.scielo.org.mx/scielo.php?script=sci_arttext\&pid=S018526982013000100006\&lng=es\&tlng=es.

Torrano, G. (2004). El aprendizaje autorregulado: presente y futuro de la investigación. Electronic Journal of Research in Educational Psychology, 13-14-15. Obtenido de https://www.redalyc.org/pdf/2931/293152878002.pdf

Valdés, \& Pujol. (2012). Autorregulación y rendimiento académico en la transición secundaria-universidad. Redalyc, 2 - 3 . Obtenido de hhttps://biblat.unam.mx/es/revista/revista-latinoamericana-de-ciencias-socialesninez-y-juventud/articulo/autorregulacion-y-rendimiento-academico-en-latransicion-secundariauniversidad

Valiente-Barroso, C., Suárez-Riveiro, J., \& Martínez-Vicente, M. (2020). Autorregulación del aprendizaje, estrés escolar y rendimiento académico. European Journal of Education and Psychology, 161-176. doi:https://doi.org/10.30552/ejep.v13i2.358

Valiente-Barroso, C., Suárez-Riveiro, J., \& Martínez-Vicente, M. (2020). Autorregulación del aprendizaje, estrés escolar y rendimiento académico. European Journal of Education and Psychology, 13(2), 161-176). doi:https://doi.org/10.30552/ejep.v13i2.358

Valle, Rodríguez, Núñez, \& Cabanach, G. (2010). Motivación y Aprendizaje Autorregulado. Redalyc, $6 . \quad$ Obtenido de https://www.redalyc.org/pdf/284/28420640010.pdf

Vargas, M. E. (2002). El aprendizaje de la ciencia y de la información científica en la educación superior. Revista cientifica española, 3-15. Obtenido de https://revistas.um.es/analesdoc/article/view/2151

Vega López, N. A., Bañales Faz, G., Reyna Valladares, A., \& Pérez Amaro, E. (2014). Enseñanza de estrategias para la comprensión de textos expositivos con alumnos de sexto grado de primaria. Scielo, 1047-1068. Obtenido de 
http://www.scielo.org.mx/scielo.php?script=sci_arttext\&pid=S1405-

$66662014000400003 \& \operatorname{lng}=$ es\&tlng=es.

Villardón, L., \& Yániz, C. (2011). LA AUTOGESTIÓN DEL APRENDIZAJE Y LA AUTONOMÍA E INICIATIVA PERSONAL. doi:isbn: 978-84-8458-354-7

Villavicencio, L. (2004). El aprendizaje autónomo en la educación a distancia. LATINEDUCA, 1-11. Obtenido de https://seminario-taller-apa-miceatic.webnode.com.ar/files/2000000143bf4e3cefb/APRENDIZAJE_AUTONOMO_A_DISTANCIA.pdf

Xhemajli, A. (2016). The role of the teacher in interactive teaching. International Journal of Cognitive Research in Science, Engineering and Education, 4(1), 31-38. doi:doi:10.5937/IJCRSEE1601031X

Yubero, S., \& Larrañaga, E. (2015). Lectura y universidad: hábitos lectores de los estudiantes universitarios de España y Portugal. Profesional De La Información, 24(6), 717-723.

Obtenido

de https://revista.profesionaldelainformacion.com/index.php/EPI/article/view/epi.2 015.nov.03

Zulma, L. (2006). El Aprendizaje Autorregulado. Buenos Aires: Ediciones Novedades Educativas. doi:ISBN-10:987-538-160-8

\section{UL Ciencia}


PARA CITAR EL ARTÍCULO INDEXADO.

Núñez Naranjo, A. F., Becerra García, E. B., \& Olalla Pardo, V. E. (2021). Autogestión del aprendizaje: Revisión de la literatura. Explorador Digital, 5(2), 6-22. https://doi.org/10.33262/exploradordigital.v5i2.1649

\section{Liencia}

El artículo que se publica es de exclusiva responsabilidad de los autores y no necesariamente reflejan el pensamiento de la Revista Explorador Digital.

El artículo queda en propiedad de la revista y, por tanto, su publicación parcial y/o total en otro medio tiene que ser autorizado por el director de la Revista Explorador Digital.
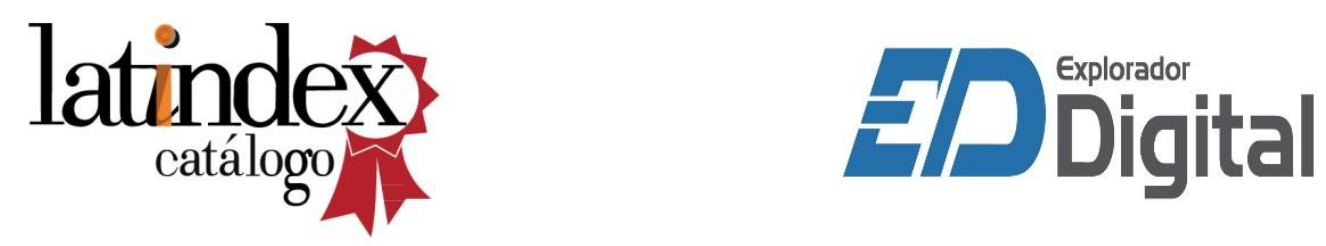